\title{
METHOD TO ESTABLISH THE OPTIMAL VARIANT OF A TECHNICAL PROJECT Octavian-Ioan BOGDAN
}

\author{
The "Nicolae Bălcescu" Land Forces Academy, Sibiu, Romania, \\ obogdan@armyacademy.ro
}

\begin{abstract}
The design phase is the most important step of the lifecycle of a technical system. If the manufacture process of any product is based on high quality technical documentation, then there is a chance to achieve a very good product. The paper aims to provide a method for determining the optimal variant of a technical project based on certain technical, economic conditions or terms of use imposed in the early stages of design.
\end{abstract}

Keywords: technical system, technical project, design alternative, objective, weight, optimal variant.

\section{Introduction}

The design process of a technical system is part of the conception phase, the most important stage of its life cycle. All the other stages of the life cycle are conditioned by performance resulting from the design. For this reason, a good solution adopted during the design phase leads to operational performance and to good economic behavior, regardless of the type of product. The conception process, being purely creative, involves: documentation, information, selection and formulation of the solutions, followed by the selection of the project variant that best meets the project specifications, formulated on the basis of the requirements of the customers. Any designer who has to choose between more alternatives for solving the required problem, is faced a dilemma: Which is the best variant? The specialized literature presents several methods to help determine the best alternative when many other possible alternatives are present. Some of the methods, simpler ones, take into account as a criterion of choice, the performance levels, while others which are, more elaborate, take into account several objectives to be achieved, objectives that are assigned certain weights, based on which it a utility score is calculated, and which is used afterwards as a criterion for choosing the optimal variant [1].

In work [1], Nigel Cross presents the weighted objectives method as a method used to establish the optimal variant of design when several solutions for the problem are possible. This requires the establishment of a set of criteria, which has to be based on the objectives of the design, which must be set at the beginning of the respective stage. During the next stages of the life cycle, these objectives may be modified because as the process progresses there may appear new constraints that could not be taken into account during the initial phase. In general, the objectives are established taking into account the technical and economic factors, the requirements of the customers, the functional safety requirements, the quality requirements, etc. 
Some of the objectives may contain design requirements and some of these may be more important than the others. In this case, assigning weights is absolutely necessary, each weight being established in accordance with the importance of the objective to which it is assigned. The respective weights may be set in the form of discrete sets or as intervals. After establishing the weights, an objective tree is created. It enables division of the objectives into sub-objectives, which in their turn are assigned the fractions of the weight established for the objective they are part of. The last stage of the method consists in determining the utility scores of the project variants, scores used in choosing the optimal variant.

\section{Presentation of the method}

We consider:

$\boldsymbol{V}=\left\{\boldsymbol{v}_{\boldsymbol{i}} \mid \boldsymbol{i}=\overline{\mathbf{1}, \boldsymbol{n}} ; \boldsymbol{n} \in \mathbb{N}\right\}$ the set of the variants of the technical project;

$\boldsymbol{C}=\left\{\boldsymbol{c}_{\boldsymbol{j}} \mid \boldsymbol{j}=\overline{\mathbf{1}, \boldsymbol{m}} ; \boldsymbol{m} \in \mathbb{N}\right\}$ the set of the required conditions of the project;

$\boldsymbol{q}_{\boldsymbol{i}}$ the coefficient of the variant $\boldsymbol{v}_{\boldsymbol{i}}, \boldsymbol{i}=\overline{\mathbf{1}, \boldsymbol{n}}$; $\boldsymbol{w}_{\boldsymbol{j}}$ the weight of the condition $\boldsymbol{c}_{\boldsymbol{j}}, \boldsymbol{j}=\overline{\mathbf{1}, \boldsymbol{m}}$;

The weights of the conditions are established by a group of experts [2], each expert giving a weight for each condition $\boldsymbol{c}_{\boldsymbol{j}}$. Consequently, a certain expert $\boldsymbol{k},(\boldsymbol{k}=\overline{\mathbf{1}, \boldsymbol{p}})$ will assign the weights of the following set $\left(w_{k 1}, w_{k 2}, w_{k 3} \ldots, w_{k m}\right)$, $(\boldsymbol{k}=\overline{\mathbf{1}, \boldsymbol{p}})$, meeting the following condition $\sum_{j=1}^{m} \boldsymbol{w}_{k j}=1$. As a result, the values of the weightsof the $\boldsymbol{p}$ sets can be grouped into the matrix of the weights assigned by the experts [3]:

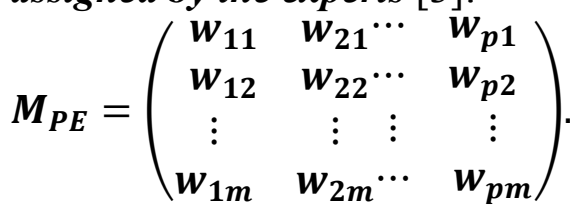

Generally, in a group of experts, their opinions related to a particular issue may be different. We consider that, as far as the design variants are considered, the $\boldsymbol{p}$ experts have different levels of competence. As a result, we assume that a "meta-expert" [2] is authorized to allocate to expert $\boldsymbol{k},(\boldsymbol{k}=$ $\overline{\mathbf{1}, \boldsymbol{p}})$ a level of the competence $\boldsymbol{g}_{\boldsymbol{k}},(\boldsymbol{k}=$ $\overline{\mathbf{1}, \boldsymbol{p}})$, on condition that $\sum_{k=1}^{p} \boldsymbol{g}_{\boldsymbol{k}}=\mathbf{1}$. Thus, the expert competences matrix results, [3].

$M_{C}=\left(\begin{array}{c}g_{1} \\ g_{2} \\ \vdots \\ g_{p}\end{array}\right)$.

We consider the matrix of the weights of the conditions [3]:

$M_{P C}=\left(\begin{array}{c}w_{1} \\ w_{2} \\ \vdots \\ w_{m}\end{array}\right)$

Relation (4) expresses the fact that the elements of matrix $\boldsymbol{M}_{\boldsymbol{P C}}$ are obtained by aggregation as explained in [2] and [3].

$M_{P C}=M_{P E} \times M_{C}$.

There result the following expressions of the weights of the conditions:

$$
\left\{\begin{array}{c}
w_{1}=w_{11} g_{1}+w_{21} g_{2}+\cdots+w_{p 1} g_{p} \\
w_{2}=w_{12} g_{1}+w_{22} g_{2}+\cdots+w_{p 2} g_{p} \\
\vdots \\
w_{m}=w_{1 m} g_{1}+w_{2 m} g_{2}+\cdots+w_{p m} g_{p}
\end{array}\right.
$$

Depending on the specifications into which the requirements of the customers are transposed [4] related to the fulfillment of conditions $\boldsymbol{c}_{\boldsymbol{j}},(\boldsymbol{j}=\overline{\mathbf{1}, \boldsymbol{m}})$, we define a new parameter $\boldsymbol{y}_{\boldsymbol{i} \boldsymbol{j}}$, which expresses the importance of condition $\boldsymbol{c}_{\boldsymbol{j}},(\boldsymbol{j}=\overline{\mathbf{1}, \boldsymbol{m}})$ in variant $\boldsymbol{v}_{\boldsymbol{i}},(\boldsymbol{i}=\overline{\mathbf{1}, \boldsymbol{n}})$ and which reflects the extent to which this condition was respected in the design of the respective variant. $\boldsymbol{y}_{\boldsymbol{i} \boldsymbol{j}}$ has values in the range $[\mathbf{0 , 1}]$. In this case, the importance matrix is:

$M_{I}=\left(\begin{array}{cccc}y_{11} & y_{12} \cdots & y_{1 m} \\ y_{21} & y_{22} & \cdots & y_{2 m} \\ \vdots & \vdots & \vdots & \vdots \\ y_{n 1} & y_{n 2} & \cdots & y_{n m}\end{array}\right)$.

We define the matrix of the coefficients of the variants:

$M_{V}=\left(\begin{array}{c}q_{1} \\ q_{2} \\ \vdots \\ q_{n}\end{array}\right)$

The matrix equation for determining these is:

$M_{V}=M_{I} \times M_{P C}$,

by means of which we can deduce the coefficients of each variant: 


$$
\left\{\begin{array}{c}
q_{1}=y_{11} w_{1}+y_{12} w_{2}+\cdots+y_{1 m} w_{m} \\
q_{2}=y_{21} w_{1}+y_{22} w_{2}+\cdots+y_{2 m} w_{m} \\
\vdots \\
q_{n}=y_{n 1} w_{1}+y_{n 2} w_{2}+\cdots+y_{n m} w_{m}
\end{array}\right.
$$

The optimal variant of the project is established by means of the following relationship:

$\boldsymbol{q}_{\text {optim }}=\max \left(\boldsymbol{q}_{1}, \boldsymbol{q}_{2}, \ldots, \boldsymbol{q}_{n}\right)$.

3. Case study

We consider the case of a technical project for which three variants were made. Thus is resulted that the set of the variants is $\boldsymbol{V}=\left\{\boldsymbol{v}_{\boldsymbol{i}} \mid \boldsymbol{i}=\overline{\mathbf{1 , 3}}\right\}$. In order to choose the optimal variant are take into consideration four conditions imposed on the project, the set of the conditions being $C=\left\{c_{j} \mid j=\overline{\mathbf{1}, 4}\right\}$.

These conditions are:

$\boldsymbol{c}_{\boldsymbol{1}}$-the technical performance of the designed product;

$\boldsymbol{c}_{2}$-the costs for the production of the product;

$\boldsymbol{c}_{3}$-the predicted reliability of the product; $\boldsymbol{c}_{\mathbf{4}}$ - the ease of use.

In order to establish the weights of the conditions the opinions of five experts are required. The values of the weights they assigned the conditions are the following:

-expert 1:

-expert 2:

$$
\begin{aligned}
& w_{11}=0,36 ; w_{12}=0,22 ; \\
& w_{13}=0,28 ; w_{14}=0,14 ;
\end{aligned}
$$

-expert 3:

$$
\begin{aligned}
& w_{21}=0,23 ; w_{22}=0,34 \\
& w_{23}=0,16 ; w_{24}=0,27
\end{aligned}
$$

$$
\begin{aligned}
& w_{31}=0,14 ; w_{32}=0,41 ; \\
& w_{33}=0,32 ; w_{34}=0,13 ;
\end{aligned}
$$

-expert 4:

$$
w_{41}=0,15 ; w_{42}=0,17
$$

-expert 5:

$$
\begin{aligned}
& w_{51}=0,25 ; w_{52}=0,25 ; \\
& w_{53}=0,25 ; w_{54}=0,25
\end{aligned}
$$

The matrix of the weights assigned by the experts is the following:

$M_{P E}=$

$$
=\left(\begin{array}{llll}
\mathbf{0}, 36 & 0,23 & 0,140,15 & 0,25 \\
0,22 & 0,34 & 0,410,17 & 0,25 \\
0,28 & 0,16 & 0,320,17 & 0,25 \\
0,14 & 0,27 & 0,130,51 & 0,25
\end{array}\right) .
$$

"The meta-expert" [2] grants the following levels of competence to the five experts: -for the expert 1: $\boldsymbol{g}_{\mathbf{1}}=\mathbf{0 , 2 3}$;

-for the expert $2: \boldsymbol{g}_{\mathbf{2}}=\mathbf{0}, \mathbf{1 7}$;

-for the expert $3: \boldsymbol{g}_{\mathbf{3}}=\mathbf{0}, \mathbf{2 2}$;

-for the expert $4: \boldsymbol{g}_{\mathbf{4}}=\mathbf{0}, \mathbf{2 5}$;

-for the expert $5: \boldsymbol{g}_{\mathbf{5}}=\mathbf{0}, \mathbf{1 3}$.

The matrix of the competences of the experts is:

$M_{C}=\left(\begin{array}{l}0,23 \\ 0,17 \\ 0,22 \\ 0,25 \\ 0,13\end{array}\right)$.

After solving the equation $\boldsymbol{M}_{P C}=\boldsymbol{M}_{\boldsymbol{P E}} \times \boldsymbol{M}_{\boldsymbol{C}}$, the following solution is obtained:

$M_{P C}=\left(\begin{array}{l}0,223 \\ 0,274 \\ 0,236 \\ 0,267\end{array}\right)$.

This means that the weights of the conditions are:

$$
\begin{gathered}
w_{1}=0,223 ; w_{2}=0,274 \\
w_{3}=0,236 ; w_{14}=0,267
\end{gathered}
$$

We consider the following importance matrix:

$$
M_{I}=\left(\begin{array}{lll}
0,6 & 0,8 & 0,40,5 \\
0,7 & 0,7 & 0,80,4 \\
0,5 & 0,9 & 0,70,6
\end{array}\right)
$$

By solving the equation $\boldsymbol{M}_{\boldsymbol{V}}=\boldsymbol{M}_{\boldsymbol{I}} \times \boldsymbol{M}_{\boldsymbol{P C}}$, there result the following values of the coefficients corresponding to the variants:

$q_{1}=0,581 ; q_{2}=0,643 ; q_{3}=0,684$.

There results:

$q_{\text {optim }}=\max \left(\boldsymbol{q}_{1}, \boldsymbol{q}_{2}, \boldsymbol{q}_{3}\right)=$

$=\max (0,581 ; 0,643 ; 0,684)=0,684$

In conclusion, the optimal variant of the project is $\boldsymbol{v}_{\mathbf{3}}$.

\section{Conclusions}

This paper introduces a new method of determining the optimal variant of a technical project, which is very simple to implement. The more difficult problem consists in choosing the relevant conditions that the respective project must meet and the correct quantification of the different parameters, that are taken into consideration so that the result should be edifying. In relation to the weighted objectives method, this method is easier to understand and to apply and we consider the results comparable. A problem that may hinder the application is finding the group 
of experts and of "the meta-expert" [2] that

to competently decide on the project and to assign weights to the conditions as accurately as possible.

\section{References}

[1] Cross, N., Engineering Design Methods. Strategies for Product Design, pp. 140-147, Wiley, 2000.

[2] Kruse, R., Schwecke, E., Heinsohn, J., Uncertainty an Vagueness in Knowledge Based Systems. Numerical Methods, pp.79, Springer-Verlag Berlin Heidelberg, 1991.

[3] Bogdan, O.I., Fuzzy Method to Establish the Impact of Human Errors on Man-Machine Systems, The $20^{\text {th }}$ International Conference The Knowledge-Based Organization, Conference Proceedings 3, pp. 17-22, The „Nicolae Bălcescu” Land Forces Academy, 1214 June, 2014.

[4] Bogdan, O.I., Managementul calităţii totale, pp. 40, „Nicolae Bălcescu” Land Forces Academy Publishing House, Sibiu, 2010. 